\title{
Surgical Results of Patients with Myelopathy due to Ossification of the Ligamentum Flavum with Ossification of the Posterior Longitudinal Ligament or a Vertebral Fracture at the Same Level of the Thoracic Spine: A Retrospective Comparative Study
}

\author{
Yuji Kasukawa, Naohisa Miyakoshi, Michio Hongo, Yoshinori Ishikawa, \\ Daisuke Kudo, Ryota Kimura, Yuichi Ono, Jumpei Iida, Chiaki Sato, Yoichi Shimada \\ Department of Orthopedic Surgery, Akita University Graduate School of Medicine, Akita, Japan
}

\begin{abstract}
Study Design: Retrospective and comparative study.
Purpose: We assessed surgical treatment outcomes in patients with thoracic myelopathy due to ossification of the ligamentum flavum (OLF), and OLF combined with ossification of the posterior longitudinal ligament (OPLL) or vertebral fracture (VF) at the same level.

Overview of Literature: OLF and OPLL cause severe thoracic myelopathy. Osteoporotic VF commonly occurs at the thoracolumbar junction. There have been no investigations of thoracic myelopathy due to OLF and VF.

Methods: Forty patients were divided among three groups: the OLF group ( $n=23)$ : myelopathy due to OLF, the OLF+OPLL group ( $n=12)$ : myelopathy due to OLF and OPLL, and the OLF+VF group ( $n=5)$ : myelopathy due to OLF and VF. We recorded OLF, OPLL, and VF sites and operative procedures. Each patient's neurological status, according to the Japanese Orthopaedic Association (JOA) score, and walking ability were evaluated pre- and postoperatively.

Results: Patients in the OLF+OPLL group were significantly younger than those in the other two groups. The preoperative JOA score was significantly lower in the OLF+VF than OLF group. The final JOA score was significantly lower in the OLF+VF than OLF and OLF+OPLL groups. The JOA score recovery rate was significantly lower in the OLF+VF than OLF group. Final walking ability was significantly worse in the OLF+OPLL and OLF+VF groups than in the OLF group and significantly worse in the OLF+VF than OLF+OPLL group. Conclusions: Thoracic myelopathy due to OLF+VF occurs primarily in older females, who also exhibit worse preoperative and postoperative neurological status, and worse walking ability, than patients with thoracic myelopathy due to OLF or OLF+OPLL.
\end{abstract}

Keywords: Ligamentum flavum ossification; Posterior longitudinal ligament ossification; Osteoporotic fracture; Compression fracture; Myelopathy

Received Oct 27, 2018; Revised Dec 19, 2018; Accepted Jan 10, 2019

Corresponding author: Yuji Kasukawa

Department of Orthopedic Surgery, Akita University School of Medicine, 1-1-1 Hondo, Akita 010-8543, Japan

Tel: +81-18-884-6148, Fax: +81-18-836-2617, E-mail: kasukawa@doc.med.akita-u.ac.jp 


\section{Introduction}

Ossification of the thoracic ligamentum flavum (OLF) reportedly affects $12 \%$ to $36 \%$ of Japanese people, based on computed tomography (CT) findings [1]. The lower third of the thoracic spine, especially T10 to T11, are the most commonly affected thoracic levels in patients with OLF [1]. The second most commonly affected levels are T4 and T5 [1]. However, when OLF occurs at the same level as ossification of the posterior longitudinal ligament (OPLL) of the thoracic spine, the combined ossification sometimes causes severe thoracic myelopathy. Aizawa et al. [2] reported that the rate of concurrent OPLL and OLF was $7.6 \%$. Sato and Aizawa [3] analyzed 265 patients who underwent surgery for thoracic myelopathy and reported OLF combined with OPLL in 9\%. Hirabayashi [4] recommended early surgical treatment for patients with combined OLF and OPLL at the same level.

Osteoporotic vertebral fractures (VFs) occur most frequently at the level of the thoracolumbar junction [5], and delayed union or non-union of an osteoporotic VF causes a neurological deficit [6]. OLF and VF may appear as a common lesion of the lower thoracic spine. We have treated several patients with thoracic myelopathy due to osteoporotic VF and OLF at the same level.

We were unable to find any previous reports that described thoracic myelopathy due to a VF and OLF at the same level, or that even peripherally addressed these patients' surgical results. The aim of this study was therefore to assess the clinical results of surgical treatment of thoracic myelopathy due to OLF and OLF combined with either OPLL or osteoporotic VF.

\section{Materials and Methods}

\section{Patients}

This was a retrospective observational study of patients who were diagnosed with, and surgically treated for, thoracic myelopathy due to OLF from January 2007 to December 2016 at Akita Uniersity Hospital, Akita, Japan. A diagnosis of OLF-related thoracic myelopathy was based on clinical, radiological, and pathological evaluations. Included participants underwent surgery for treatment of myelopathy due to thoracic OLF, with a postoperative follow-up period of $\geq 1$ year. In total, 40 patients were enrolled in the study and divided among three groups: the OLF group ( $n=23)$ : thoracic myelopathy due to OLF alone, the OLF+OPLL group $(n=12)$ : thoracic myelopathy due to OLF and OPLL at the same level (at least one level), and the OLF+VF group $(n=5)$ : thoracic myelopathy due to OLF and a clinical VF at the same level.

The retrospective research in the present study was approved by the Institutional Review Board of Akita University Graduate School of Medicine (IRB approval no., 1879) and was performed in accordance with the ethical standards established in the Declaration of Helsinki. Informed consent was obtained from each patient in this study.

\section{Evaluations}

1) Clinical and neurological features

We reviewed the medical records of all patients and recorded their ages, sex, and time from symptom-onset to surgery. The Japanese Orthopaedic Association (JOA) score for thoracic myelopathy was used to assess each patient's neurological status preoperatively and at the final follow-up [7]. The recovery rate (\%) was calculated according to Hirabayashi et al. [8] as follows: (postoperative [final follow-up] JOA score-preoperative JOA score)/ (11-preoperative JOA score $) \times 100$. In addition, pre- and postoperative walking ability, operative procedure, other operations for cervical or lumbar lesions, operation time, and estimated intraoperative blood loss were evaluated.

\section{2) Radiological features}

Plain radiographic images, $\mathrm{CT}$, and magnetic resonance imaging scans were reviewed. The levels of OLF, OPLL, and VF in the upper (T1-T4), middle (T5-T8), and lower (T9-T12) thoracic spine were recorded on CT images. OLF was classified into five types based on the range and morphological features of the ossification depicted on the preoperative CT scans at the narrowest spinal level. These types included lateral, extended, enlarged, fused, and tuberous [9].

\section{3) Representative radiological cases}

Fig. 1 shows a typical case in the OLF group. OLF was present at levels T9 to T10 and levels T10 to T11 on sagittal T1-weighted images (T1WI) and T2-weighted images (T2WI), respectively (Fig. 1A, B), and the OLF was compressing the spinal cord on the axial T1WI and T2WI at levels T10 to T11, respectively (Fig. 1D, E). Sagittal and axial CT images after myelography demonstrated extend- 

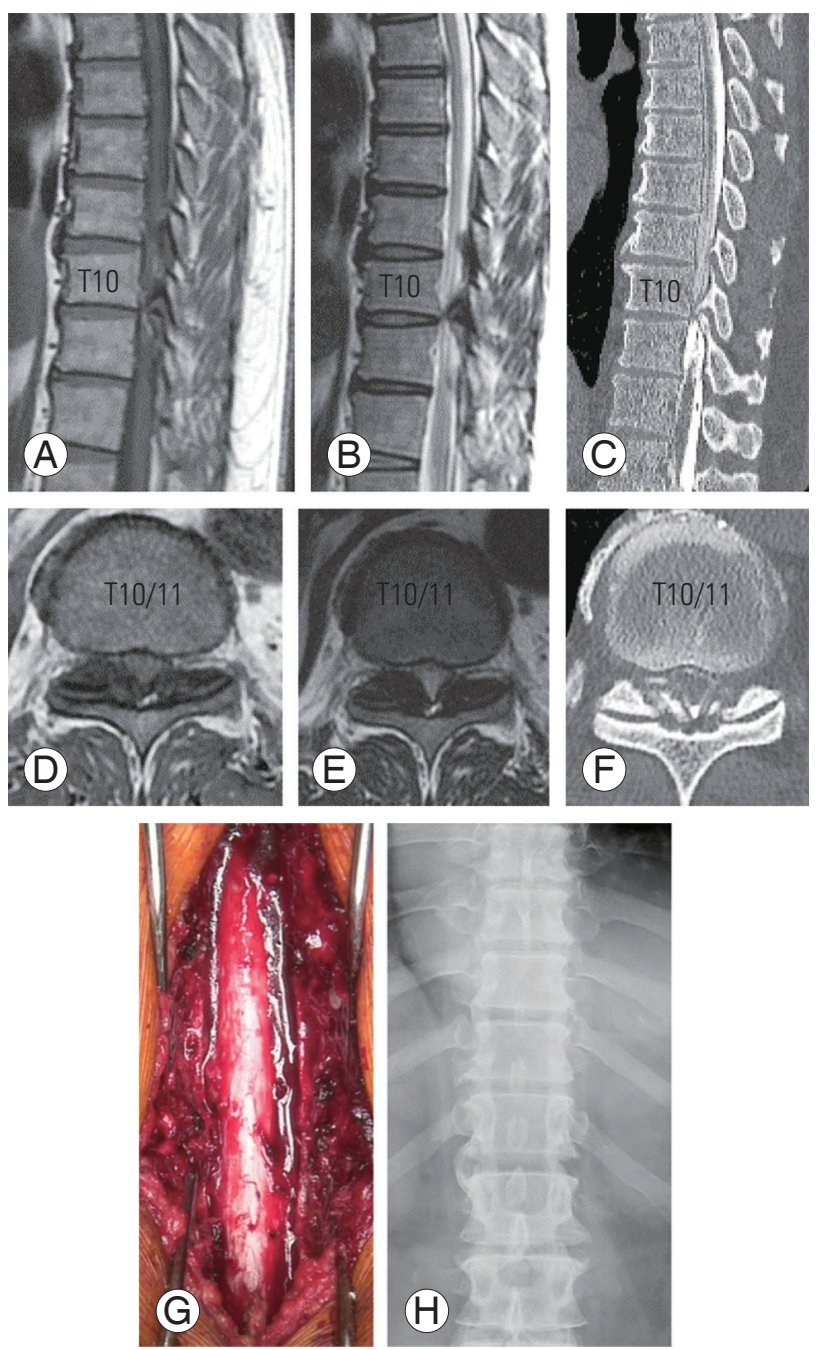

Fig. 1. Sagittal (A) T1WI and (B) T2WI show low-intensity OLF at levels T10 to T11 and levels T9 to T10. (C) Sagittal post-myelographic CT shows OLF compressing the dural sac. Axial (D) T1WI and (E) T2WI at levels T10 to T11 show OLF with the low-intensity, compressed spinal cord. (F) Post-myelographic axial CT image shows extended OLF at levels T10 to T11, causing severe stenosis and spinal cord compression. (G) Decompressive wide laminectomy and removal of OLF from T9 to L1 was performed. (H) Postoperative plain radiography shows the laminectomy from T9 to L1. T1WI, T1-weighted magnetic resonance imaging; T2WI, T2-weighted magnetic resonance imaging; OLF, ossification of the ligamentum flavum; CT, computed tomography.

ed OLF at levels T10 to T11, respectively (Fig. 1C, F).

Fig. 2 shows a case in the OLF+OPLL group. OLF and OPLL were present at multiple levels of the thoracic spine (Fig. 2A). Extended-type OLF (Fig. 2E) and beak-type OPLL (Fig. 2F) were present at levels T1 to T2, and tuberous-type OLF (Fig. 2G) and small OPLL were present at levels T11 to T12. These severe OLF+OPLL types caused major compression of the spinal cord at levels $\mathrm{T} 1$ to $\mathrm{T} 2$ (Fig. 2B) and T11 to T12 (Fig. 2C, D).
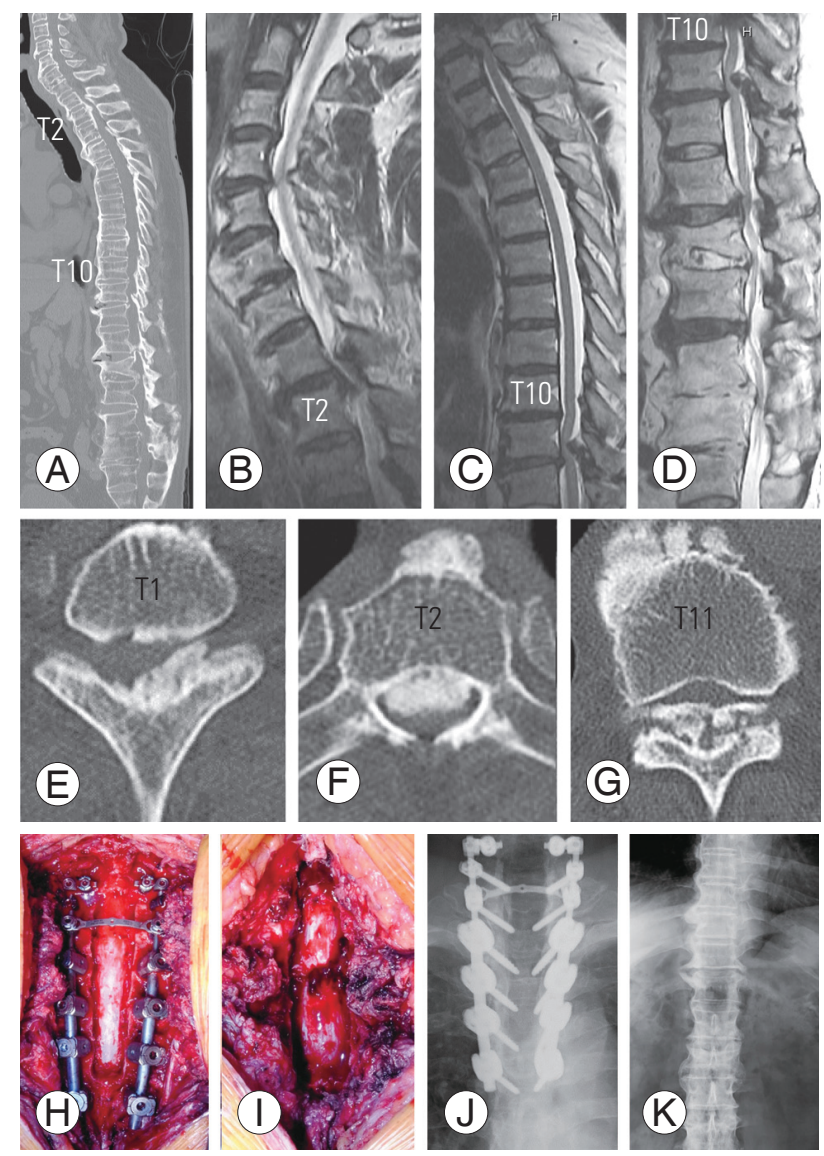

Fig. 2. (A) Sagittal CT image from the cervical to lumbar spine indicates beaked-type OPLL at levels T2 to T3 and small OPLLs at levels T8 to T9 and T11 to T12. Sagittal T2-weighted magnetic resonance imaging of the (B) cervical and upper thoracic spine, (C) thoracic spine, and (D) lumbar spine indicates (B) OLF at levels T1 to T2 and T3 to $\mathrm{T} 4$ and OPLL at levels $\mathrm{T} 1$ to $\mathrm{T} 2$ and (C, D) OLF at levels T11 to T12 compressing the spinal cord with (D) lumbar spinal stenosis. Axial CT images reveal unilateral (E) extended OLF at levels T1 to T2, (F) OPLL at $\mathrm{T} 1$ to $\mathrm{T} 2$, and (G) tuberous OLF at T11 to T12. (H) Wide laminectomy and removal of OLF from C7 to T4 was performed following posterior fusion and stabilization from C6 to T5 using pedicle screws and rods. (I) Simultaneous decompression by wide laminectomy and removal of tuberous OLF was performed from T10 to T12. Postoperative plain radiography shows $(\mathrm{J})$ decompression and posterior fusion of the cervicothoracic lesion with instruments and $(\mathbf{K})$ decompression by resection of OLF at the lower thoracic lesion. CT, computed tomography; OPLL, ossification of the posterior longitudinal ligament; OLF, ossification of the ligamentum flavum.

Fig. 3 shows a case of OLF+VF. A clinical VF was present at T12 with low intensity on T1WI (Fig. 3A) and high intensity on T2WI (Fig. 3B). CT showed the VF at T12 (Fig. 3C) and enlarged OLF at T11 to T12 (Fig. 3D) and T12 to L1 (Fig. 3C, F) and on the posterior wall of the fractured vertebral body (Fig. 3E) compressing the spinal cord. 


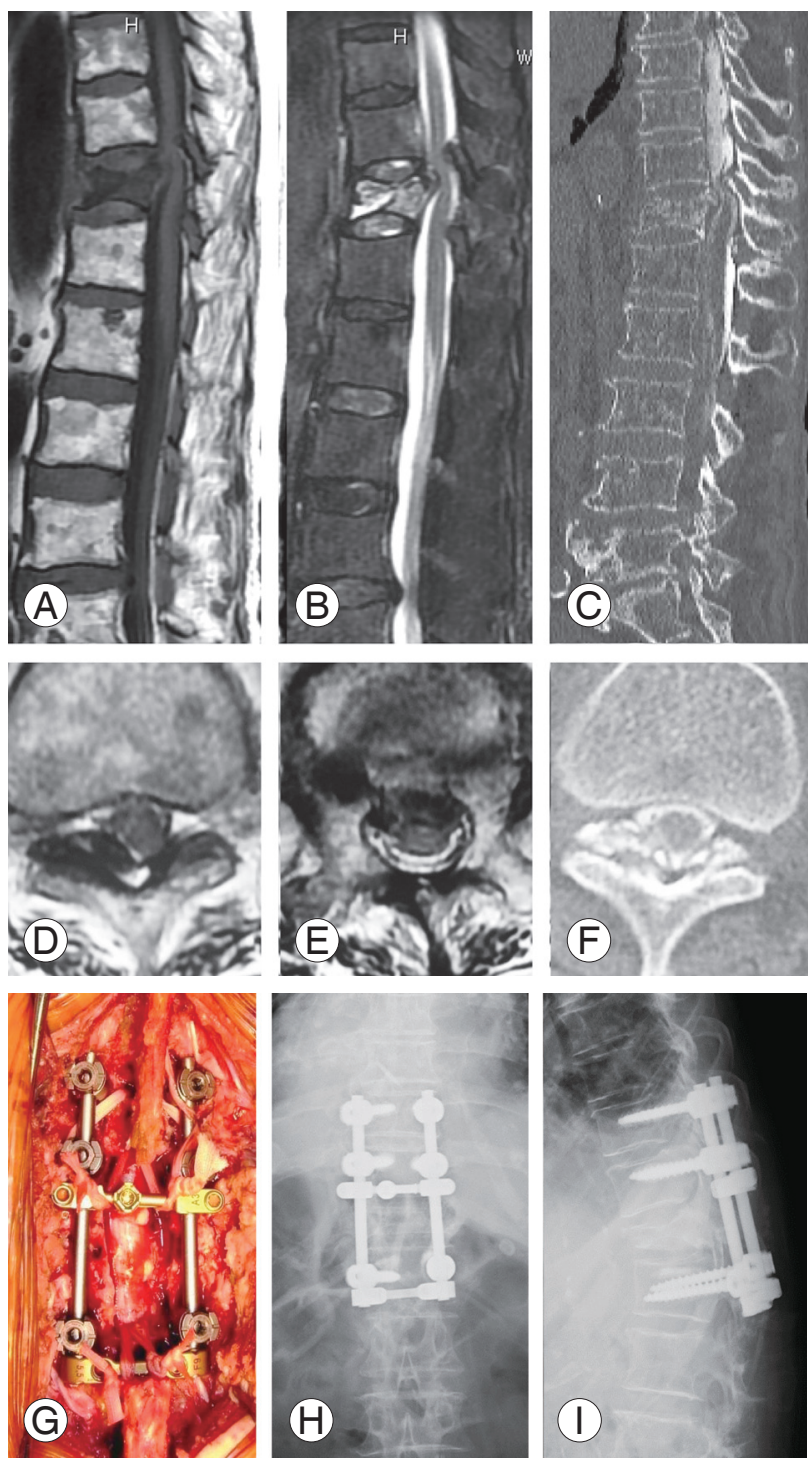

Fig. 3. Sagittal (A) T1WI and (B) fat-suppressed T2WI show a T12 clinical vertebral fracture with (A) low intensity and (B) high intensity, respectively, as well as OLF at levels T11 to T12 and T12 to L1 compressing the spinal cord. (C) Sagittal post-myelographic CT shows severe stenosis at levels T11 to T12 on the posterior wall of the fractured vertebral body and OLF. Axial (D) T1WI and (E) T2WI show (D) stenosis with low-intensity OLF and (E) the posterior wall of the fractured vertebral body of T12. (f) Axial post-myelographic CT shows an enlarged OLF compressing the dural sac. (G) Wide laminectomy and OLF removal was performed after posterior instrumentation and stabilization using pedicle screws and rods, with additional stabilization using sublaminar tape at the remaining cranial and caudal laminae. Postoperative (H) anteroposterior and (I) lateral plain radiography show posterior decompression and fusion at the level of the OLF and vertebral fracture. T1WI, T1-weighted magnetic resonance imaging; T2WI, T1-weighted magnetic resonance imaging; OLF, ossification of the ligamentum flavum; CT, computed tomography.
4) Operative procedures

All patients underwent surgery with electrophysiological monitoring of spinal cord activity. Posterior decompressive wide laminectomies were performed. Spinal levels that required decompression were identified based on the patients' preoperative neurological status and imaging findings. Decompressive laminectomies, which consisted of removing the laminae and OLF with the medial half of the facet joint, were performed. Decompression was undertaken using a high-speed surgical drill (Medtronic, Minneapolis, MN, USA) (Fig. 1G, H).

If the patient had OLF and OPLL at the same level, we performed posterior fusion with a pedicle screws-and-rod system. Usually, we performed fusion three levels above and three below the OPLL and OLF to stabilize and repair the kyphosis. Briefly, we inserted pedicle screws and connected a rod on one side in situ before performing the laminectomy to avoid neurological aggravation immediately after laminectomy due to alterations of the spinal alignment. After total laminectomy, bilateral rods were connected under spinal cord monitoring, which was used to observe the amplitude and latency of the waves [10] (Fig. 2H-K).

Patients with OLF and VF underwent laminectomy and posterior fusion using the same procedure as for those with OLF and OPLL. For OLF+VF, however, in addition to the laminectomy and posterior fusion, sublaminar tape was used on the remaining cranial and caudal laminae to avoid back-out of instruments (Fig. 3G). Fusion was performed two levels above and one or two levels below the fractured vertebra (Fig. 3H, I). In two cases, we diagnosed the fractured vertebra with non-union, and we performed vertebroplasty with a hydroxyapatite block inserted into the fractured vertebra [11].

\section{Statistical analyses}

Bartlett test results indicated that no data except age were normally-distributed. Hence, results are expressed as medians (interquartile range) or mean \pm standard deviation. Differences between the groups were analyzed using Fisher's exact test for nominal variables, the Kruskal-Wallis test for non-parametric continuous variables, or analysis of variance for parametric continuous variables, followed by the Bonferroni or Mann-Whitney $U$-test method for multiple comparisons. Differences with a value of $p<0.05$ were considered statistically significant. All statistical 
Table 1. Patients' demographic and background data

\begin{tabular}{|c|c|c|c|c|}
\hline Characteristic & $\operatorname{OLF}(n=23)$ & OLF+OPLL (n=12) & $O L F+V F(n=5)$ & $p$-value \\
\hline Sex (male:female) & $17: 6$ & $6: 6$ & $0: 5$ & $0.007^{a)}$ \\
\hline Age (yr) & $66.1 \pm 13.8$ & $49.8 \pm 11.6^{b)}$ & $78.8 \pm 4.7^{c)}$ & $<0.001^{d)}$ \\
\hline Preoperative duration of symptoms (mo) & $3.0(2.0-5.5)$ & $3.0(2.0-4.3)$ & $1.0(1.0-2.0)$ & $0.209^{e)}$ \\
\hline Follow-up periods (mo) & $30.0(14.5-43.0)$ & $60.0(28.5-75.0)$ & $12.0(12.0-12.0)$ & $0.057^{\mathrm{el}}$ \\
\hline
\end{tabular}

Values are presented as number, mean \pm standard deviation, or median (interquartile range).

OLF, ossification of the ligamentum flavum; OPLL, ossification of the posterior longitudinal ligament; VF, vertebral fracture.

${ }^{a}$ B By Fisher's exact test. ${ }^{b /} p<0.01$ vs. OLF. ${ }^{c)} p<0.01$ vs. OLF+OPLL (Bonferroni test). ${ }^{d)} B y$ analysis of variance. ${ }^{\text {el}}$ By Kruskal-Wallis test.

analyses were performed using EZR software (Jichi Medical University, Jichi Medical University, Saitama, Japan) [12].

\section{Results}

\section{Patients' backgrounds}

The patients in the OLF+OPLL group (average age, 49.8 years) were significantly younger than those in the OLF group (average age, 66.1 years; $p<0.01$ ) and $\mathrm{OLF}+\mathrm{VF}$ group (average age, 78.8 years; $p<0.01$ ). All patients reported a preoperative symptom duration of 1 to 3 months, with no significant differences among the three groups. There were no statistically significant differences in the follow-up periods among the three groups, although the follow-up period was shorter in the OLF+VF group than in the other groups (Table 1).

\section{Level or type of ossification of the ligamentum flavum, ossification of the posterior longitudinal ligament, and vertebral fracture}

OLF mostly affected lower levels (T9-T12) in the OLF and OLF+VF groups. There were no significant differences in OLF type among the three groups, although the extended type was most common in the OLF group, the fused type in the OLF+OPLL group, and the enlarged type in the OLF+VF group (Table 2). Concomitant OPLL was most common in upper levels (T1-T4), and VF was most common in the lower thoracic spine (T10-T12).

\section{Operative characteristics}

Laminectomy at the level of the OLF was performed in $87 \%$ of patients in the OLF group. Laminectomy and pos-
Table 2. Level or type of OLF, OPLL, and VF

\begin{tabular}{|c|c|c|c|c|}
\hline Characteristic & $\begin{array}{c}\text { OLF } \\
(n=23)\end{array}$ & $\begin{array}{c}\text { OLF+OPLL } \\
(n=12)\end{array}$ & $\begin{array}{c}\mathrm{OLF}+V F \\
(n=5)\end{array}$ & $p$-value \\
\hline Level of OLF & & & & $0.004^{\mathrm{al}}$ \\
\hline Upper (T1-4) & 0 & 2 & 0 & \\
\hline Middle (T5-8) & 0 & 3 & 0 & \\
\hline Lower (T9-12) & 19 & 2 & 5 & \\
\hline Upper and middle & 1 & 1 & 0 & \\
\hline Upper and lower & 1 & 2 & 0 & \\
\hline Upper to lower & 0 & 1 & 0 & \\
\hline Middle to lower & 2 & 1 & 0 & \\
\hline Type of OLF & & & & $0.290^{\text {a) }}$ \\
\hline Lateral & 2 & 0 & 0 & \\
\hline Extended & 10 & 2 & 1 & \\
\hline Enlarged & 5 & 2 & 3 & \\
\hline Fused & 4 & 5 & 1 & \\
\hline Tuberous & 2 & 3 & 0 & \\
\hline \multicolumn{5}{|l|}{ Level of OPLL } \\
\hline Upper & - & 7 & - & \\
\hline Middle & - & 0 & - & \\
\hline Lower & - & 1 & - & \\
\hline Upper to middle & - & 3 & - & \\
\hline Upper to lower & - & 1 & - & \\
\hline \multicolumn{5}{|l|}{ Level of fracture } \\
\hline $\mathrm{T} 10$ & - & - & 1 & \\
\hline T11 & - & - & 1 & \\
\hline $\mathrm{T} 12$ & - & - & 3 & \\
\hline
\end{tabular}

OLF, ossification of the ligamentum flavum; OPLL, ossification of the posterior longitudinal ligament; VF, vertebral fracture.

alBy Fisher's exact test.

terior fusion were performed significantly more often in the OLF+OPLL and OLF+VF groups and were combined with vertebroplasty using hydroxyapatite in two patients 
Table 3. Operative characteristics

\begin{tabular}{|c|c|c|c|c|}
\hline Characteristic & OLF (N=23) & OLF+OPLL (N=12) & $O L F+V F(N=5)$ & $p$-value \\
\hline Operation & & & & $<0.001^{\text {a) }}$ \\
\hline LMN & 20 & $0^{b)}$ & $0^{b)}$ & \\
\hline LMN and fusion & 3 & 12 & 3 & \\
\hline LMN+vertebroplasty and fusion & 0 & 0 & 2 & \\
\hline Operative time (min) & $253.0(187.5-307.0)$ & $428.0(324.0-697.5)^{c)}$ & $302.0(281.0-321.0)$ & $0.001^{\mathrm{d})}$ \\
\hline Estimated blood loss (mL) & $143.0(80.5-294.0)$ & $543.0(245.0-1,031.0)^{c)}$ & $282.0(192.0-659.0)$ & $0.011^{\mathrm{d})}$ \\
\hline Additional operation for cervical or lumbar lesion & & & & $0.383^{\mathrm{a})}$ \\
\hline Cervical laminoplasty & 6 & 4 & 0 & \\
\hline Lumbar decompression & 2 & 0 & 0 & \\
\hline
\end{tabular}

Values are presented as number or median (interquartile range).

OLF, ossification of the ligamentum flavum; OPLL, ossification of the posterior longitudinal ligament; VF, vertebral fracture; LMN, laminectomy.

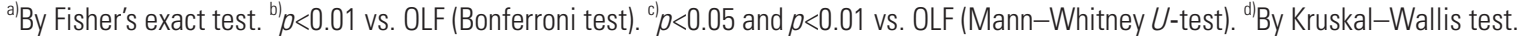

Table 4. Preoperative and postoperative clinical and neurological results

\begin{tabular}{|c|c|c|c|c|}
\hline Variable & $\mathrm{OLF}(\mathrm{N}=23)$ & OLF+OPLL (N=12) & $\mathrm{OLF}+\mathrm{VF}(\mathrm{N}=5)$ & $p$-value \\
\hline Preoperative walking ability & & & & $0.028^{\text {a) }}$ \\
\hline Without support & 2 & 2 & 0 & \\
\hline With support & 13 & 4 & 0 & \\
\hline Impossible (wheelchair) & 8 & 6 & 5 & \\
\hline Final walking ability & & & & $<0.001^{\text {a) }}$ \\
\hline Without support & 16 & $3^{\text {b) }}$ & $0^{\mathrm{b}, \mathrm{cl}}$ & \\
\hline With support & 7 & 7 & 0 & \\
\hline Impossible (wheelchair) & 0 & 2 & 5 & \\
\hline \multicolumn{5}{|l|}{ JOA score } \\
\hline Preoperative score & $5.5(4.3-6.3)$ & $3.5(3.0-6.0)$ & $3.0(3.0-3.0)^{d}$ & $0.033^{e)}$ \\
\hline Final score & $9.0(8.0-9.5)$ & $7.8(5.8-8.6)$ & $4.0(3.0-5.0)^{d, f)}$ & $<0.001^{\mathrm{e})}$ \\
\hline Recovery rate & $62.5(40.0-76.0)$ & $38.2(19.2-70.0)$ & $14.3(12.5-20.0)^{d)}$ & $0.005^{e)}$ \\
\hline
\end{tabular}

Values are presented as median (interquartile range).

OLF, ossification of the ligamentum flavum; OPLL, ossification of the posterior longitudinal ligament; VF, vertebral fracture; JOA, Japanese Orthopaedic Association.

${ }^{a}$ By Fisher's exact test. ${ }^{b} p<0.05$ and $p<0.01$ vs. OLF (by Bonferroni test). ${ }^{c \mid} p<0.05$ vs. OLF+OPLL (by Bonferroni test). ${ }^{d)} p<0.05$ and $p<0.01$ vs. OLF (by Mann-Whitney $U$-test). ${ }^{e}$ By Kruskal-Wallis test. ${ }^{f} p<0.05$ vs. OLF+OPLL (by Mann-Whitney $U$-test).

in the OLF+VF group $(p<0.01)$ (Table 3). The operative time and estimated blood loss were significantly greater in the OLF+OPLL than OLF group $(p<0.01$ and $p<0.05$, respectively). There were no significant differences in the operative time or estimated blood loss between the OLF+OPLL and OLF+VF groups. An additional cervical laminoplasty was performed in six patients in the OLF group and four patients in the OLF+OPLL group. Two patients in the OLF group required additional lumbar decompression.

\section{Preoperative and postoperative clinical and neuro- logical outcomes}

Preoperatively, 91\% (21/23) of the OLF group, 83\% (10/12) of the OLF+OPLL group, and $100 \%$ (5/5) of the OLF+VF group were unable to walk (i.e., patients were unable to walk [wheelchair-bound] or required support with a cane or wheelchair) (Table 4). Final walking ability was significantly worse in the OLF+OPLL and OLF+VF groups than in the OLF group ( $p<0.05$ and $p<0.01$, respectively), and it 
was significantly worse in the OLF+VF than OLF+OPLL group $(p<0.05)$.

The preoperative JOA score was significantly lower in the OLF+VF than OLF group $(p<0.05)$ (Table 4$)$. The final JOA score was significantly lower in the OLF+VF group than in either the OLF or OLF+OPLL group $(p<0.01$ and $p<0.05$, respectively). JOA score recovery was significantly lower in the OLF+VF than OLF group $(p<0.01)$. However, there was no significant difference in the recovery rate of the JOA score between the OLF+VF group (14.3\%) and the OLF+OPLL group (38.2\%).

\section{Discussion}

\section{Worst preoperative and postoperative clinical results in patients with ossification of the ligamentum fla- vum and vertebral fracture}

Patients with OLF+VF at the same level had the worst preoperative thoracic myelopathy symptoms and postoperative clinical results. The recovery rate after surgery was also significantly poorer in the OLF+VF, compared to the OLF group. The OLF+VF group consisted of only women, who were significantly older than patients in the OLF+OPLL group. The OLF type and the preoperative period were not significantly different among the groups.

The older age of patients in the OLF+VF group might have influenced these patients' poor pre- and postoperative clinical results and walking ability, as it related to thoracic myelopathy. Furthermore, all patients in the $\mathrm{OLF}+\mathrm{VF}$ group were female. The background of the older, female-only OLF+VF group might have contributed to the poor pre- and postoperative clinical results and walking ability. In addition to their older age, their osteoporotic VFs showed non-union or instability at the various fracture sites. VF instability at the OLF level might have caused severe spinal cord injury. Ando et al. [13] reported that discontinuous ossification of the anterior longitudinal ligament combined with OLF causes more severe symptoms preoperatively and poorer surgical outcomes. Instability at the OLF level may cause severe myelopathic symptoms and worse surgical results in patients with OLF-related thoracic myelopathy.

\section{Other factors related to clinical results of ossification of the ligamentum flavum-related thoracic myelopathy}

Previous reports have also identified factors related to the clinical results after surgical treatment of OLF-related thoracic myelopathy, including the preoperative severity of the myelopathy [14], dural adhesions of OLF [15], a concomitant lumbar spinal lesion [16], impaired sense of the joint position in the big toe [17], and an intramedullary signal change on T2WI $[16,17]$. In our previous study, preoperative symptom duration was the most important predictor of long-term surgery-related outcomes in patients with OLF-related thoracic myelopathy [9]. The type of OLF, the presence of dural adhesions, and the need for concomitant surgery for coexistent cervical or lumbar lesions do not influence the long-term postoperative prognosis [9]. Onishi et al. [18] reported that simultaneous OPLL and OLF in the mid-thoracic spine was associated with relatively poor recovery. In the present study, patients with OLF and OPLL had significantly worse postoperative walking ability than those with OLF alone. Thus, the combination of OLF and OPLL at the same level is a significant causal factor for worse surgical outcomes.

\section{Surgical treatment of ossification of the ligamentum flavum and vertebral fracture}

OLF-related thoracic myelopathy is treated by decompressive surgery, such as wide laminectomy and OLF removal $[9,14]$. In the present series, decompressive surgery for the patients with thoracic myelopathy due to OLF alone produced satisfactory results. When VF occurs at the level of the OLF, however, posterior decompressive surgery should be avoided because it could worsen fracture site instability. We believe that fusion should be performed with decompressive surgery.

Many surgical procedures to treat clinical or non-union VFs have been reported, such as anterior decompression and fusion [19], balloon kyphoplasty [20], vertebroplasty with polymethylmethacrylate [21] or hydroxyapatite [11] blocks, and posterior-approach vertebral replacement with rectangular parallelepiped cages [22]. In one study, the rate of perioperative complications associated with the posterior approach using spinal instrumentation for osteoporotic VF reportedly ranged from $16.1 \%$ to $22.9 \%$ in patients with primary or secondary osteoporosis [23]. We usually perform posterior surgery for patients with 
osteoporotic VFs. Patients with OLF require posterior decompression. Thus, posterior decompression and fusion surgery is an appropriate procedure for patients with thoracic myelopathy due to OLF+VF.

\section{Surgical treatment of ossification of the ligamentum flavum+ossification of the posterior longitudinal ligament}

A variety of surgical procedures have been developed to treat thoracic OPLL in patients with OLF+OPLL-related thoracic myelopathy, including posterior decompressive laminectomy or laminoplasty [24], posterior decompression and fusion [24], two-stage posterior and anterior decompression [25], and circumferential decompression via a posterior approach $[24,25]$. Li et al. [10] reported that posterior decompression with instrumented fusion resulted in a considerable degree of neurological recovery, despite anterior impingement of the spinal cord by the remaining OPLL. In the present study, posterior decompression and fusion using instrumentation for patients with thoracic myelopathy due to OLF+OPLL at the same level provided relief comparable with that after posterior decompressive surgeries for patients with myelopathy due to OLF alone.

\section{Limitations}

This study had several limitations. First, it was a retrospective study and thus lacks prospective validation. In addition, these retrospective data are based on outcomes after various types of surgeries, such as decompression only, decompression and fusion at single or multiple levels, and decompression and fusion with vertebroplasty. Second, the follow-up periods were different among the three groups, although the differences were not statistically significant. Third, the patients in the OLF+VF group were older than those in the OLF-alone group, and patients in the OLF+VF group had more-impaired walking abilities than patients in the other groups. Long followup periods were difficult for the patients in the OLF+VF group. Finally, the sample size of the OLF+VF group $(n=5)$ was much smaller than the other groups.

\section{Conclusions}

Patients with thoracic myelopathy caused by OLF com- bined with VF had worse preoperative and postoperative neurological status and walking abilities than those with thoracic myelopathy due to OLF alone or OLF+OPLL. Posterior decompression and fusion with vertebroplasty is an acceptable option for surgical treatment of thoracic myelopathy due to OLF+VF.

\section{Conflict of Interest}

No potential conflict of interest relevant to this article was reported.

\section{Acknowledgments}

We thank the medical staff at our institute for helping with the data collection. We thank Nancy Schatken, BS, MT (ASCP) and Angela Morben, DVM, ELS from Edanz Group (http://www.edanzediting.com/ac) for editing a draft of this manuscript.

\section{ORCID}

Yuji Kasukawa: https://orcid.org/0000-0001-7008-675X

Naohisa Miyakoshi: https://orcid.org/0000-0001-5175-3350

Michio Hongo: https://orcid.org/0000-0003-1929-2448

Yoshinori Ishikawa: https://orcid.org/0000-0001-6697-4527

Daisuke Kudo: https://orcid.org/0000-0001-8626-1057

Ryota Kimura: https://orcid.org/0000-0002-4855-3283

Yuichi Ono: https://orcid.org/0000-0001-9250-457X

Jumpei Iida: https://orcid.org/0000-0001-5378-8047

Chiaki Sato: https://orcid.org/0000-0002-3728-8154

Yoichi Shimada: https://orcid.org/0000-0002-6523-3249

\section{Author Contributions}

Conception and design: $\mathrm{YK}$; data acquisition: $\mathrm{YK}, \mathrm{MH}$, YI, DK; analysis of data: YK, NM; drafting of the manuscript: YK; critical revision and supervision: NM, YS; and administrative support: MH, YI, DK, RK, YO, JI, CS.

\section{References}

1. Mori K, Kasahara T, Mimura T, et al. Prevalence, distribution, and morphology of thoracic ossification of the yellow ligament in Japanese: results of CTbased cross-sectional study. Spine (Phila Pa 1976) 2013;38:E1216-22. 
2. Aizawa T, Sato T, Sasaki H, et al. Results of surgical treatment for thoracic myelopathy: minimum 2-year follow-up study in 132 patients. J Neurosurg Spine 2007;7:13-20.

3. Sato T, Aizawa T. Current status of thoracic myelopathy. Sekitsui Sekizui (Spine Spinal Cord) 2009;22:13641.

4. Hirabayashi S. Ossification of the ligamentum flavum. Spine Surg Relat Res 2017;1:158-63.

5. Hongo M, Miyakoshi N, Shimada Y, Sinaki M. Association of spinal curve deformity and back extensor strength in elderly women with osteoporosis in Japan and the United States. Osteoporos Int 2012;23:102934.

6. Baba H, Maezawa Y, Kamitani K, Furusawa N, Imura S, Tomita K. Osteoporotic vertebral collapse with late neurological complications. Paraplegia 1995;33:2819.

7. Sato T, Kokubun S, Tanaka Y, Ishii Y. Thoracic myelopathy in the Japanese: epidemiological and clinical observations on the cases in Miyagi Prefecture. Tohoku J Exp Med 1998;184:1-11.

8. Hirabayashi K, Miyakawa J, Satomi K, Maruyama T, Wakano K. Operative results and postoperative progression of ossification among patients with ossification of cervical posterior longitudinal ligament. Spine (Phila Pa 1976) 1981;6:354-64.

9. Miyakoshi N, Shimada Y, Suzuki T, et al. Factors related to long-term outcome after decompressive surgery for ossification of the ligamentum flavum of the thoracic spine. J Neurosurg 2003;99:251-6.

10. Li M, Meng H, Du J, Tao H, Luo Z, Wang Z. Management of thoracic myelopathy caused by ossification of the posterior longitudinal ligament combined with ossification of the ligamentum flavum-a retrospective study. Spine J 2012;12:1093-102.

11. Oshima M, Matsuzaki H, Tokuhashi Y, Okawa A. Evaluation of biomechanical and histological features of vertebrae following vertebroplasty using hydroxyapatite blocks. Orthopedics 2010;33:89-93.

12. Kanda Y. Investigation of the freely available easy-touse software 'EZR' for medical statistics. Bone Marrow Transplant 2013;48:452-8.

13. Ando K, Imagama $S$, Ito $Z$, et al. Predictive factors for a poor surgical outcome with thoracic ossification of the ligamentum flavum by multivariate analysis: a multicenter study. Spine (Phila Pa 1976)
2013;38:E748-54.

14. Aizawa T, Sato T, Sasaki H, Kusakabe T, Morozumi N, Kokubun S. Thoracic myelopathy caused by ossification of the ligamentum flavum: clinical features and surgical results in the Japanese population. J Neurosurg Spine 2006;5:514-9.

15. Li Z, Ren D, Zhao Y, et al. Clinical characteristics and surgical outcome of thoracic myelopathy caused by ossification of the ligamentum flavum: a retrospective analysis of 85 cases. Spinal Cord 2016;54:188-96.

16. Matsumoto $\mathrm{Y}$, Harimaya K, Doi T, et al. Clinical characteristics and surgical outcome of the symptomatic ossification of ligamentum flavum at the thoracic level with combined lumbar spinal stenosis. Arch Orthop Trauma Surg 2012;132:465-70.

17. Kawaguchi Y, Yasuda T, Seki S, et al. Variables affecting postsurgical prognosis of thoracic myelopathy caused by ossification of the ligamentum flavum. Spine J 2013;13:1095-107.

18. Onishi E, Sano H, Matsushita M. Surgical treatment for thoracic myelopathy due to simultaneous ossification of the posterior longitudinal ligament and ligamentum flavum at the same level. Clin Spine Surg 2016;29:E389-95.

19. Kanayama M, Ishida T, Hashimoto T, et al. Role of major spine surgery using Kaneda anterior instrumentation for osteoporotic vertebral collapse. J Spinal Disord Tech 2010;23:53-6.

20. Franck H, Boszczyk BM, Bierschneider M, Jaksche H. Interdisciplinary approach to balloon kyphoplasty in the treatment of osteoporotic vertebral compression fractures. Eur Spine J 2003;12:S163-7.

21. Heini PF, Walchli B, Berlemann U. Percutaneous transpedicular vertebroplasty with PMMA: operative technique and early results: a prospective study for the treatment of osteoporotic compression fractures. Eur Spine J 2000;9:445-50.

22. Suzuki T, Abe E, Miyakoshi N, et al. Posterior-approach vertebral replacement with rectangular parallelepiped cages (PAVREC) for the treatment of osteoporotic vertebral collapse with neurological deficits. J Spinal Disord Tech 2013;26:E170-6.

23. Miyakoshi N, Kobayashi T, Suzuki T, Kikuchi K, Kasukawa Y, Shimada Y. Perioperative medical complications after posterior approach spinal instrumentation surgery for osteoporotic vertebral collapse: a comparative study in patients with primary osteopo- 
rosis and those with secondary osteoporosis. Asian Spine J 2017;11:756-62.

24. Yamazaki M, Mochizuki M, Ikeda Y, et al. Clinical results of surgery for thoracic myelopathy caused by ossification of the posterior longitudinal ligament: operative indication of posterior decompression with instrumented fusion. Spine (Phila Pa 1976) 2006;31:1452-60.
25. Tomita K, Kawahara N, Baba H, Kikuchi Y, Nishimura H. Circumspinal decompression for thoracic myelopathy due to combined ossification of the posterior longitudinal ligament and ligamentum flavum. Spine (Phila Pa 1976) 1990;15:1114-20. 